\title{
I 56 Pulmonary vein imaging by 3D non-contrast, free breathing SSFP MR angiography; a novel technique
}

\author{
Aparna Singhal ${ }^{* 1}$, Anderanik Tomasian ${ }^{1}$, Alex Sassani ${ }^{1}$, Vibhas Deshpande ${ }^{2}$, \\ Gerhard Laub' ${ }^{1}$ J Paul Finn ${ }^{1}$, Stefan Ruehm ${ }^{1}$ and Mayil S Krishnam ${ }^{1}$
}

Address: ${ }^{1}$ UCLA, Los Angeles, CA, USA and ${ }^{2}$ Siemens Medical Solutions, Los Angeles, CA, USA

* Corresponding author

from I th $^{\text {th }}$ Annual SCMR Scientific Sessions

Los Angeles, CA, USA. I-3 February 2008

Published: 22 October 2008

Journal of Cardiovascular Magnetic Resonance 2008, IO(Suppl I):A57 doi:I0.1 I86/I532-429X-10-SI-A57

This abstract is available from: http://jcmr-online.com/content/I0/SI/A57

(c) 2008 Singhal et al; licensee BioMed Central Ltd.

\section{Introduction}

Radiofrequency ablation of foci in pulmonary veins has emerged as a therapeutic option for patients with medically refractory atrial fibrillation or those unable to tolerate drugs. Preprocedural imaging is important and is being increasingly utilized to assess pulmonary venous anatomy. Contrast enhanced Magnetic Resonance Angiography (CEMRA) has been shown to be an efficient modality for assessment of pulmonary veins with excellent spatial resolution without any exposure to radiation and iodinated contrast. However, CE-MRA is operator dependant and requires patient cooperation for breath holding. Additionally, high dose gadolinium use has been recently reported to be associated with the occurrence of Nephrogenic Systemic Fibrosis (NSF) in certain patients including renal disease patients. Recently, three dimensional navigator gated free breathing selective steady-state free precession (SSFP) sequence has been employed to display non-contrast MR angiography of the heart and great vessels. The SSFP causes inherent high contrast between the blood pool and background tissues in the body due to high $\mathrm{T} 2 / \mathrm{T} 1$ ratio.

\section{Purpose}

To investigate the feasibility of non-contrast 3D SSFP MR Angiography for evaluation of pulmonary veins and to correlate the results with conventional contrast-enhanced MRA (CE-MRA).

\section{Methods}

Twenty consecutive patients (age range 30-81) with known atrial fibrillation underwent free-breathing ECGgated non-contrast SSFP MRA with non-selective radiofrequency excitation and conventional high-resolution 3D CEMRA of thorax at $1.5 \mathrm{~T}$. Two readers evaluated both datasets for vein definition (from 0 , not visualized to 3 , excellent definition), artifacts, findings, and intra-vascular signal-to-noise ratio (SNR) and contrast-to-noise ratio (CNR) in the pulmonary veins. Pulmonary vein ostial measurements were also made in both the datasets. Statistical analysis was performed using Wilcoxon test, paired ttest, and kappa co-efficient.

\section{Results}

Reader 1 and 2 graded $85(96.6 \%)$ and $81(92.1 \%)$ segments on SSFP MRA, and $84(100 \%)$ and $84(100 \%)$ segments on CE-MRA as having diagnostic or excellent definition (good inter-observer agreement, $\mathrm{k}=0.68, \mathrm{p}<$ 0.01 ). Reader 1(2) identified 14(13) and 21 (23) motion artifacts on SSFP and CE-MRA, respectively. Both readers demonstrated right middle vein $(n=8)$ on both datasets. No significant difference existed for visibility scores for each reader between the two datasets except for right inferior pulmonary vein which demonstrated lower but diagnostic visibility on SSFP datasets $(\mathrm{p}<0.05)$. The grouped mean ostial diameter on SSFP datasets was $15.8 \pm 2.7 \mathrm{~mm}$ compared to $16.3 \pm 2.6 \mathrm{~mm}$ on CE-MRA. Adequate SNR and CNR was achieved in all the veins on SSFP MRA, however, the values were significantly higher on CE-MRA for 
all segments $(P<0.05)$. Scan time for SSFP MRA ranged from 5 to 10 minutes (mean \pm standard deviation, $7 \pm 2$ minutes). Figure 1 and 2: Non contrast SSFP MRA demonstrating right and left superior pulmonary veins (A) (arrows) and right and left inferior veins (B) (arrows); LALeft Atrium. Navigator saturation band is seen on the right hemithorax as a well defined vertical bar of low signal intensity.

\section{Conclusion}

Our results demonstrate that non-contrast 3D SSFP MRA provides sufficient vascular definition and SNR and CNR for confident evaluation of pulmonary veins. This technique may be an alternative approach to CE-MRA especially in patients at increased risk for developing contrastrelated complications and those with difficulty in breath holding.

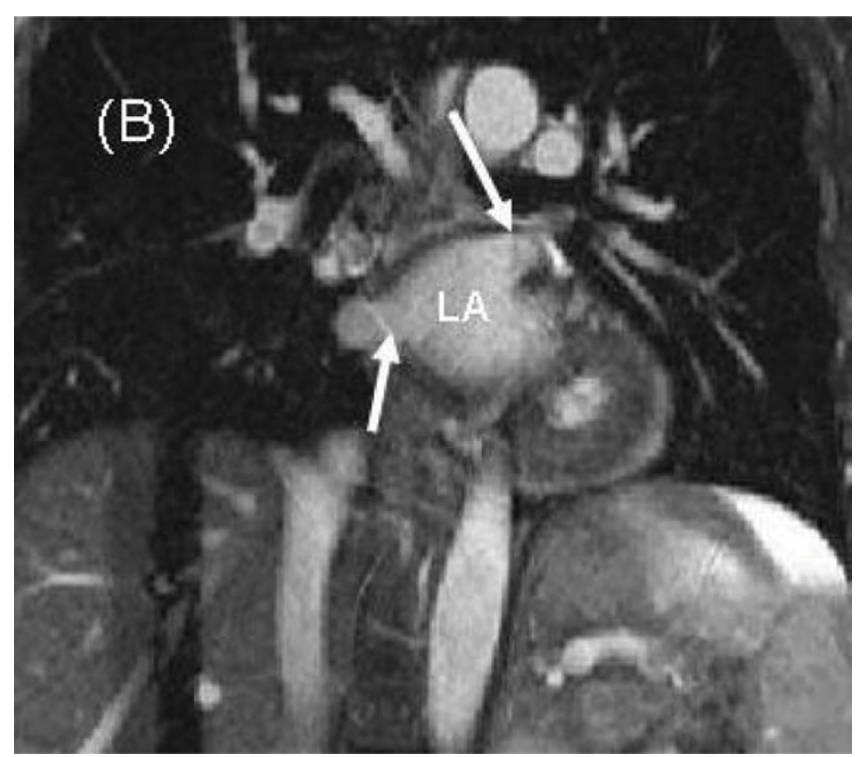

Figure I

Our aim was to investigate the feasibility of non-contrast, free breathing 3D-SSFP MR Angiography for pulmonary veins evaluation and to correlate with contrast-enhanced MRA. 3D-SSFP MRA provides sufficient vascular definition and SNR and CNR for confident evaluation of pulmonary veins. Non contrast SSFP MRA demonstrating right and left inferior veins (B) (arrows); LA: Left Atrium. Navigator saturation band is seen on the right hemithorax as a well defined vertical bar of low signal intensity.

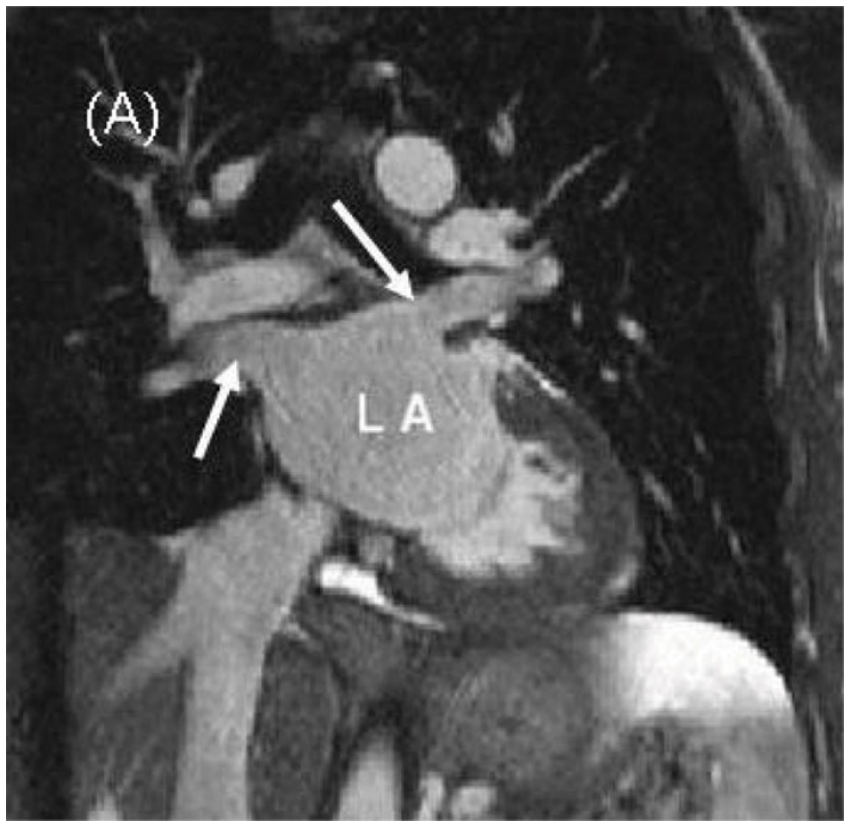

Figure 2

Non contrast SSFP MRA demonstrating right and left superior pulmonary veins (A) (arrows); LA: Left Atrium. Navigator saturation band is seen on the right hemithorax as a well defined vertical bar of low signal intensity.
Publish with Biomed Central and every scientist can read your work free of charge

"BioMed Central will be the most significant development for disseminating the results of biomedical research in our lifetime. " Sir Paul Nurse, Cancer Research UK

Your research papers will be:

- available free of charge to the entire biomedical community

- peer reviewed and published immediately upon acceptance

- cited in PubMed and archived on PubMed Central

- yours - you keep the copyright
BioMedcentral 